\title{
ESTUDIO COMPARATIVO MÉXICO - COLOMBIA PARA CONOCER LOS PRINCIPALES FACTORES DE ESTRÉS LABORAL
}

\section{COMPARATIVE STUDY MEXICO - COLOMBIA TO KNOW THE MAIN FACTORS OF WORK STRESS}

José Vili Martínez González*, Vili Aldebarán Martínez García**

*Doctor en Administración. Ajedrez Consultores. Secretario General del COLPARMEX. ORCID: https://orcid.org/0000-0002-0978-5778

**Joven Investigador para Ajedrez Consultores. ORCID: https://orcid.org/0000-0002-3901-0121

Dirección para recibir correspondencia: ajedrezconsultores@hotmail.com 
ESTUDIO COMPARATIVO MÉXICO-COLOMBIA PARA CONOCER LOS PRINCIPALES FACTORES DE ESTRÉS LABORAL

\section{RESUMEN}

El estrés se ha convertido en un problema de salud, en el ámbito laboral genera descuidos en el trabajo, disminución en la productividad, aumento en la rotación de personal y en las enfermedades mentales, esto sin mencionar el impacto económico, la Organización Internacional del Trabajo (OIT), menciona que el estrés laboral podría suponer pérdidas de entre el 0.5\% y el 3.5\% del Producto Interno Bruto (PIB) de cada país.

OBJETIVO: Conocer si los factores que generan estrés laboral son los mismos en México y Colombia.

METODOLOGÍA: Se realizó una investigación de tipo descriptiva, transeccional, utilizando Aldebarán-Test para identificar los factores principales de generación de estrés laboral, utilizando una muestra de sujeto tipo con un total de 540 considerando a México (359) y Colombia (181).

RESULTADOS: En México y Colombia los principales factores causantes de estrés resultaron ser los extraorganizacionales.

CONCLUSIONES: Los niveles de estrés grave se comportan muy similar, un gran porcentaje de la muestra está consumiendo medicamentos para ansiedad y/o depresión principalmente los que presentan estrés grave.

PALABRAS CLAVE: Estrés. Estrés laboral. Factores psicosociales. Estresores.

\section{ABSTRACT}

Stress has become a health problem, in the workplace it generates carelessness at work, decreased productivity, increased turnover of staff and mental illness, not to mention the economic impact, the International Labor Organization, mentions that the labor stress could suppose losses between $0.5 \%$ and $3.5 \%$ of the Gross Domestic Product of each country.

OBJECTIVE: To know if the factors that generate work stress are the same in Mexico and Colombia. 
ESTUDIO COMPARATIVO MÉXICO-COLOMBIA PARA CONOCER LOS PRINCIPALES FACTORES DE ESTRÉS LABORAL

MATERIAL AND METHOD: Descriptive, transectional research was carried out, using AldebaranTest to identify the main factors of generation of work stress, using a sample of subject type with a total of 540 considering Mexico (359) and Colombia (181).

RESULTS: In Mexico and Colombia the main factors causing stress turned out to be extraorganizational.

CONCLUSIONS: Severe stress levels behave very similar, a large percentage of the sample is consuming medications for anxiety and / or depression, mainly those with severe stress.

KEYWORD: Stress. Work stress. Psychosocial factors. Stressors.

\section{INTRODUCCIÓN}

Conceptualizar al estrés resulta ser muy complejo y ha sido sujeto a múltiples análisis entre diferentes expertos de diversas áreas, por ejemplo, para Papalia (1998) el estrés resulta ser una reacción fisiológica y psicológica del organismo como resultado de los requerimientos hechos sobre él; para Greenspan (1993), es una tensión orgánica o mental causada por estímulos físicos, químicos o emocionales. Para la American Psychological Association (2018) es una "experiencia emocional molesta que venga acompañada de cambios bioquímicos, fisiológicos y conductuales predecibles". En resumen, se puede considerar que el estrés es un desequilibrio del organismo con manifestaciones fisiológicas, psicológicas y bioquímicas.

El estrés resulta ser algo cotidiano y normal hasta cierto punto, ya que permite el mantenerse alerta, sin embargo, cuando esto se vuelve crónico o excesivo puede tener consecuencias en la salud, afectando el sistema cardiovascular, neuroendocrino, inmunitario y nervioso; en la tabla 1 se mencionan algunos de los trastornos más comunes que se han relacionado con el estrés. 
ESTUDIO COMPARATIVO MÉXICO-COLOMBIA PARA CONOCER LOS PRINCIPALES FACTORES DE ESTRÉS LABORAL

\section{Tabla 1}

Algunos de los trastornos más comunes relacionados con el estrés

\begin{tabular}{|c|c|}
\hline Cardiovasculares & $\begin{array}{l}\text { Arritmias cardiacas. } \\
\text { Hipertensión arterial. } \\
\text { Infarto agudo al miocardio. } \\
\text { Angina de pecho. } \\
\text { Arteriosclerosis. }\end{array}$ \\
\hline Respiratorios & $\begin{array}{l}\text { Disnea. } \\
\text { Asma. }\end{array}$ \\
\hline Digestivos & $\begin{array}{l}\text { Vómitos. } \\
\text { Regurgitación. } \\
\text { Úlceras gástricas. } \\
\text { Cólicos, diarrea o estreñimiento. } \\
\text { Gastritis y colitis. }\end{array}$ \\
\hline Sistema reproductor femenino & $\begin{array}{l}\text { Dismenorrea. } \\
\text { Amenorrea. }\end{array}$ \\
\hline Piel & $\begin{array}{l}\text { Prurito. } \\
\text { Sudoración exagerada. } \\
\text { Cambios vasculares: rubor y palidez. } \\
\text { Alopecia. }\end{array}$ \\
\hline Muscular & $\begin{array}{l}\text { Bruxismo. } \\
\text { Lumbalgia. } \\
\text { Mialgias. } \\
\text { Hipotonía muscular, (aflojamiento de las piernas) y caída por } \\
\text { estrés. }\end{array}$ \\
\hline Sistema nervioso & $\begin{array}{l}\text { Mareos o vértigos. } \\
\text { Trastornos del sueño. } \\
\text { Dificultad en la concentración. } \\
\text { Ansiedad. } \\
\text { Neurosis. }\end{array}$ \\
\hline Sistema inmunitario & Inmunosupresión. \\
\hline Sistema endocrino & $\begin{array}{l}\text { Aumento del cortisol. } \\
\text { Aumento de la prolactina. } \\
\text { Aumento de la hormona tiroidea. } \\
\text { Disminución de la hormona antidiurética. } \\
\text { Disminución de la testosterona. } \\
\text { Aumento de la glucemia plasmática. } \\
\text { Aumento de los lípidos plasmáticos. }\end{array}$ \\
\hline
\end{tabular}

Fuente: Elaboración propia.

El estrés laboral se podría definir como, la respuesta física y emocional nociva que ocurre cuando los requerimientos del trabajo no son compatibles con las capacidades, los recursos o las 

ESTRÉS LABORAL

necesidades de los trabajadores. Existen diferentes clasificaciones de los factores de riesgo de estrés laboral dependiendo del autor, entre los que se encuentran: factores ambientales, organizacionales, extra organizacionales, individuales, grupales (ver tabla 2).

Tabla 2

Factores de riesgo de estrés en el trabajo

\begin{tabular}{|c|c|}
\hline Estresor & Características \\
\hline Luz & $\begin{array}{l}\text { Resulta indispensable una adecuada iluminación para el puesto de trabajo, ya que una } \\
\text { buena iluminación se percibe como alegre y estimulante y, por tanto, relajante. }\end{array}$ \\
\hline Ruido & $\begin{array}{l}\text { El ruido excesivo y/o intermitente interfiere con la concentración y es fuente de frustración. } \\
\text { La prolongada exposición al ruido, por tanto, puede conducir a la fatiga y a la disminución } \\
\text { del desempeño. }\end{array}$ \\
\hline Temperatura & $\begin{array}{l}\text { Si la temperatura corporal y la frecuencia cardíaca se incrementan, acarrean malestares, } \\
\text { desinterés por la actividad y sed; los extremos de frío también influyen sobre los individuos, } \\
\text { afectando los niveles de energía y posiblemente disminuyendo la motivación; sin mencionar } \\
\text { las afectaciones fisiológicas que pueden provocar. }\end{array}$ \\
\hline Vibraciones & $\begin{array}{l}\text { El estrés del movimiento es precipitado por las aceleraciones giratorias de la cabeza que } \\
\text { son registradas en los canales semicirculares del oído interno. }\end{array}$ \\
\hline Aire contaminado & $\begin{array}{l}\text { Este tipo de condición atmosférica es un estresor que plantea problemas tanto físicos como } \\
\text { psicológicos la principal preocupación es la calidad del aire del sitio de trabajo. }\end{array}$ \\
\hline Radiaciones & $\begin{array}{l}\text { El daño que pudieran provocar dichas radiaciones depende de su frecuencia y energía. A } \\
\text { los efectos podemos clasificar estas radiaciones dividiendo el espectro electromagnético en } \\
\text { dos partes: radiaciones no ionizantes y radiaciones ionizantes (radiactivas). }\end{array}$ \\
\hline El diseño de la tarea & $\begin{array}{l}\text { Falta de variedad y ciclos de trabajo cortos, trabajo carente de significado, falta de } \\
\text { satisfacción. }\end{array}$ \\
\hline Estilo de dirección & $\begin{array}{l}\text { La falta de participación del empleado en la toma de decisiones y una pobre comunicación } \\
\text { en la organización. }\end{array}$ \\
\hline $\begin{array}{l}\text { Relaciones } \\
\text { interpersonales }\end{array}$ & Aislamiento social, escasa relación con los superiores, conflicto interpersonal. \\
\hline Roles de trabajo & Las expectativas de trabajo incierto, demasiada responsabilidad, información inadecuada. \\
\hline $\begin{array}{l}\text { Preocupaciones } \\
\text { profesionales }\end{array}$ & $\begin{array}{l}\text { Estancamiento profesional, promoción excesiva o insuficiente, escaso valor social del } \\
\text { trabajo. }\end{array}$ \\
\hline $\begin{array}{l}\text { Horarios y turnos en } \\
\text { el trabajo }\end{array}$ & Cambio de turnos, horarios inflexibles y/o largos o que no permiten tener vida social. \\
\hline Clima organizacional & $\begin{array}{l}\text { Una organización general deficiente puede significar frustraciones en los empleados, } \\
\text { tornándose toda la organización ineficiente. }\end{array}$ \\
\hline Económicos & Salarios bajos, inseguridad económica. \\
\hline $\begin{array}{l}\text { Influencia de } \\
\text { liderazgo }\end{array}$ & $\begin{array}{l}\text { El líder tiene la influencia más poderosa, ya que tienen autoridad y poder; la habilidad que } \\
\text { tenga el líder para aplicar este poder es decisivo para los trabajadores ya que puede } \\
\text { provocarles estrés o bien ser un facilitador. }\end{array}$ \\
\hline $\begin{array}{l}\text { Comunicación } \\
\text { inadecuada }\end{array}$ & $\begin{array}{l}\text { Comunicación pobre, bajos niveles de apoyo para la resolución de problemas y el desarrollo } \\
\text { personal. }\end{array}$ \\
\hline Extraorganizacionales & Conflicto entre el trabajo y el hogar, escaso apoyo en el hogar. \\
\hline
\end{tabular}

Fuente: Elaboración propia. 
ESTUDIO COMPARATIVO MÉXICO-COLOMBIA PARA CONOCER LOS PRINCIPALES FACTORES DE ESTRÉS LABORAL

La importancia de conocer este tema, radica en que en las últimas décadas se ha presentado un aumento el estrés relacionado con el trabajo y por ende sus consecuencias, como son:

Impacto en la salud: el estrés elevado contribuye a trastornos mentales y de comportamiento, como, el agotamiento, burnout, desgaste, ansiedad, depresión, abuso de alcohol y drogas, aumento en el índice de accidentes laborales, así como daños físicos, enfermedad cardiovascular y trastornos musculoesqueléticos (Organización Internacional del Trabajo, 2016).

Impacto económico: el estrés genera altos costos económicos tanto para la persona enferma como para sus familiares, ya que a menudo reducen su productividad tanto en el hogar como en el trabajo, pierden ingresos y además incurren en gastos de salud que puede agravar su economía, generando mayor estrés. La Organización Internacional del Trabajo (OIT), menciona que el estrés laboral podría suponer pérdidas de entre el $0.5 \%$ y el 3.5\% del Producto Interno Bruto (PIB) de cada país (Rojas, 2017).

De acuerdo al Instituto Mexicano del Seguro Social, el 75\% de los mexicanos padece de fatiga por estrés, estando por arriba de China y Estados Unidos (Instituto Mexicano del Seguro Social, 2018). En lo referente a la prevalencia en Latinoamérica tenemos que:

- Argentina, $26.7 \%$ de los trabajadores reportaron que sufrían de carga mental, considerando su trabajo excesivo.

- Brasil, $14 \%$ de las enfermedades profesionales son debidas a enfermedades mentales.

- Chile, el $27.9 \%$ de los trabajadores reportaron estrés.

- Colombia, $24.7 \%$ de hombre y 28.45 de mujeres trabajadores reportaron estrés (Organización Internacional del Trabajo, 2016).

\section{OBJETIVO}

Conocer si los factores que generan estrés laboral son los mismos en México y Colombia.

Los factores considerados son:

- Estresores del ambiente físico.

- Estresores organizacionales.

- Estresores extraorganizacionales. 
ESTUDIO COMPARATIVO MÉXICO-COLOMBIA PARA CONOCER LOS PRINCIPALES FACTORES DE ESTRÉS LABORAL

\section{MATERIAL Y MÉTODO}

Se realiza una investigación de tipo descriptiva, transeccional.

En la presentación de la encuesta se especificó el objetivo del estudio, que sería anónima, que la información sería tratada en forma confidencial y analizada en conjunto para publicaciones científicas y de difusión en congresos especializados, que en ningún caso se publicarían datos individuales. No se recogieron datos personales como nombre o algún dato que permitirá su identificación.

No existe conflicto de interés en la realización de este estudio.

Se utilizó el Aldebarán-Test (García-Valerio, 2003) para identificar los factores principales de generación de estrés laboral, este instrumento cuenta con un Alpha de 0.7933 y está constituido por tres dimensiones, las cuales son:

- Estresores del ambiente físico.

- Estresores organizacionales.

- Estresores extraorganizacionales.

Los criterios de inclusión considerados fueron personas laboralmente activas, edad de 20 a 60 años de nacionalidad mexicana y colombiana. Los criterios de exclusión considerados fueron personas menores de 19 años o mayores de 61, que no hubieran completado correctamente el test, que no estuvieran laborando o de nacionalidad diferente a la mexicana o colombiana excluyendo un total de 52 test.

Se trató de una muestra de sujeto tipo con un total de 540 cuestionarios.

Conceptualización de las variables consideradas:

- Factores ambientales: son los factores relacionados con los agentes contaminantes que se encuentran en el ambiente de trabajo (ruido, iluminación, temperatura, aire contaminado, diseño de área laboral).

- Factores organizacionales: se consideran factores como la calidad de vida en el trabajo y los conflictos relacionados con la empresa en sí, (estilo de dirección, preocupaciones profesionales, horario y turnos de trabajo económicos, clima organizacional). 
ESTUDIO COMPARATIVO MÉXICO-COLOMBIA PARA CONOCER LOS PRINCIPALES FACTORES DE ESTRÉS LABORAL

- Factores extraorganizacionales: se toman en consideración los sucesos ajenos a la organización y el tipo de conducta (dificultades familiares, competitividad).

\section{RESULTADOS}

En la figura 1, se aprecia cómo se integró el total de la muestra por 540 participantes, de los cuales 359 fueron mexicanos y 181 colombianos.

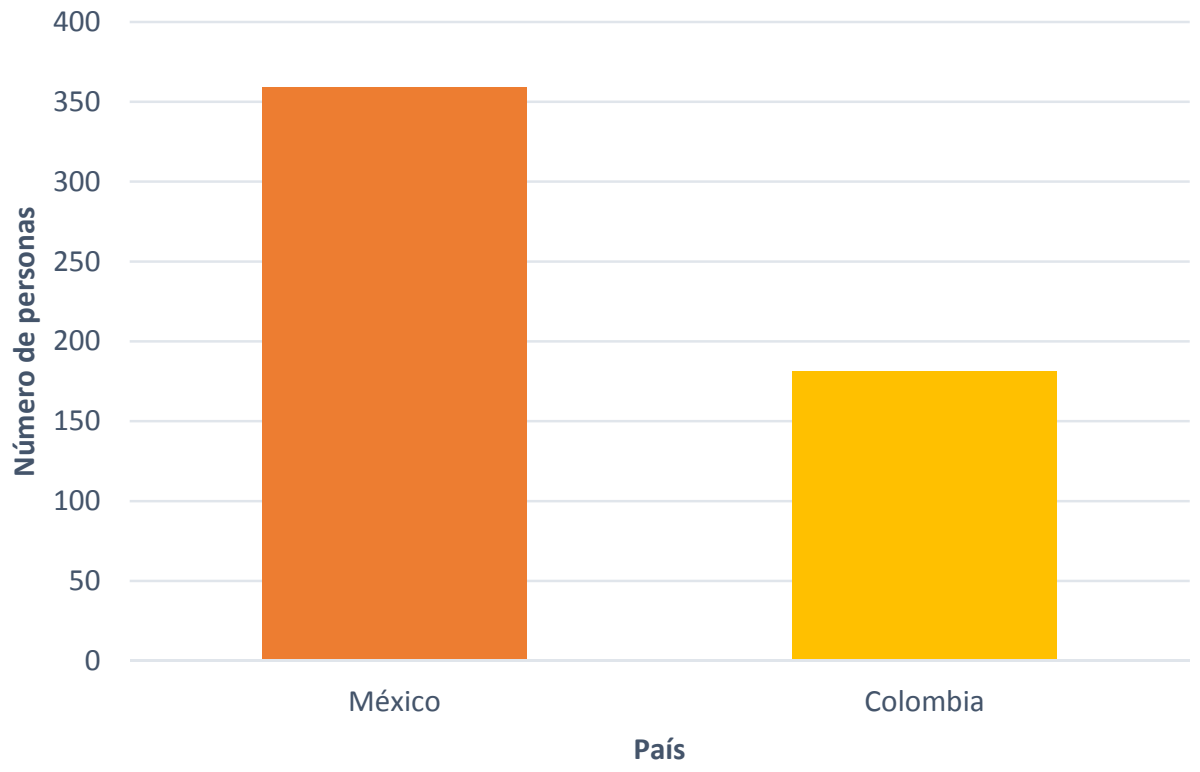

Figura 1. Total de la muestra.

Fuente: Elaboración propia. 
ESTUDIO COMPARATIVO MÉXICO-COLOMBIA PARA CONOCER LOS PRINCIPALES FACTORES DE ESTRÉS LABORAL

En la figura 2, se aprecia la composición de la muestra considerando el rango de edades.

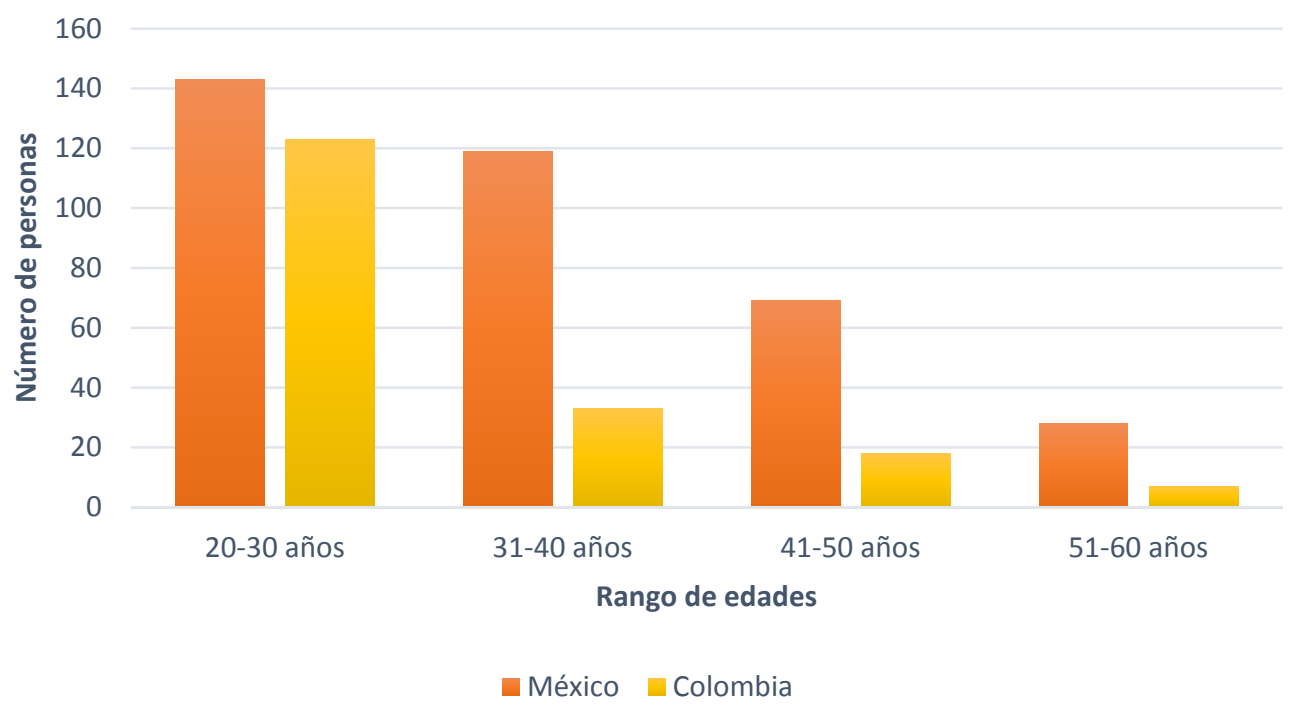

Figura 2. Rango de edades.

Fuente: Elaboración propia.

En la figura 3, es factible observar la composición de la muestra en porcentaje considerando el sexo.

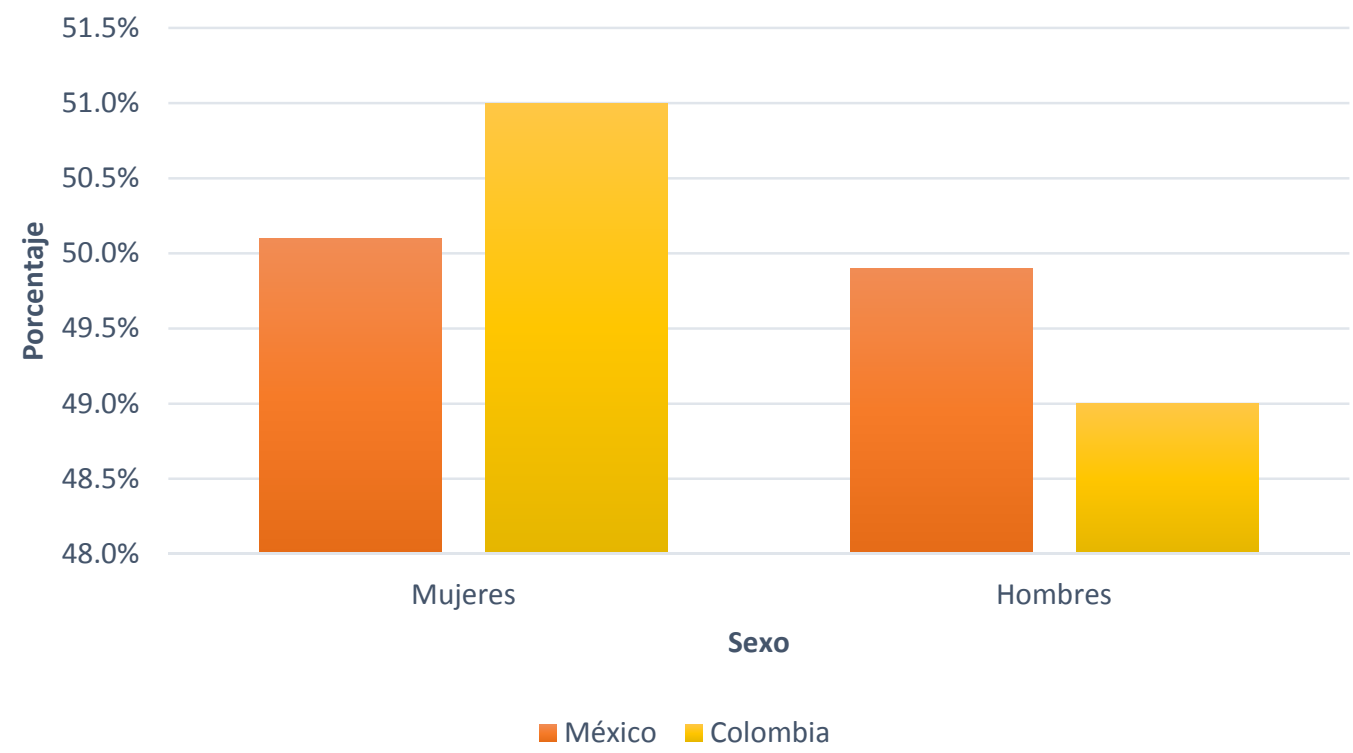

Figura 3. Sexo.

Fuente: Elaboración propia. 
ESTUDIO COMPARATIVO MÉXICO-COLOMBIA PARA CONOCER LOS PRINCIPALES FACTORES DE ESTRÉS LABORAL

En la figura 4, es posible realzar en porcentajes un comparativo de los niveles de estrés encontrados en los integrantes de la muestra; estos se dividen dependiendo de los síntomas relacionados con el estrés considerando tres niveles.

Leve. Es un nivel considerado como un estrés "cotidiano" en el cual no se presentan síntomas de afectación relacionados.

Moderado. Es el nivel de estrés donde se presentan síntomas de leves a moderados relacionados.

Grave. En este nivel se presentan múltiples y acusados síntomas relacionados.

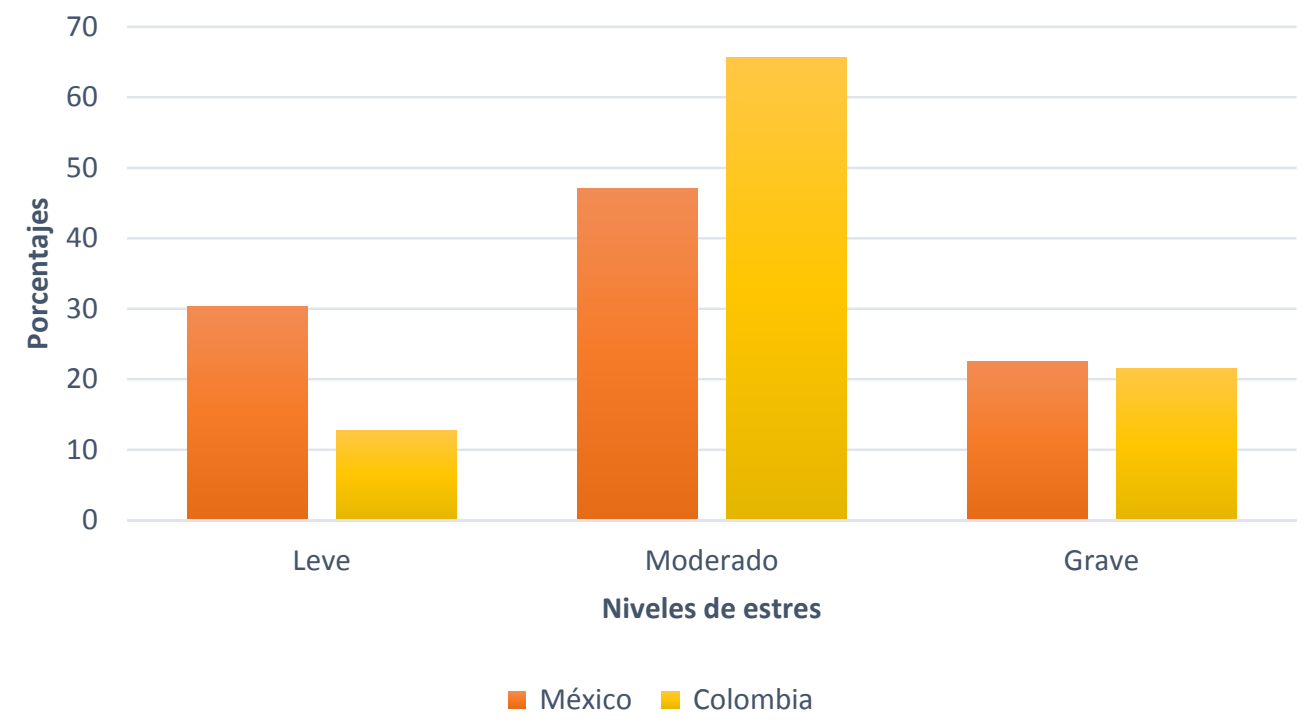

Figura 4. Nivel de estrés presentado.

Fuente: Elaboración propia.

En la figura 5, se presentan los porcentajes de los estresores referenciados por los integrantes de la muestra del tipo, encontrando que los estresores extraorganizacionales predominan en forma importante. 
ESTUDIO COMPARATIVO MÉXICO-COLOMBIA PARA CONOCER LOS PRINCIPALES FACTORES DE ESTRÉS LABORAL

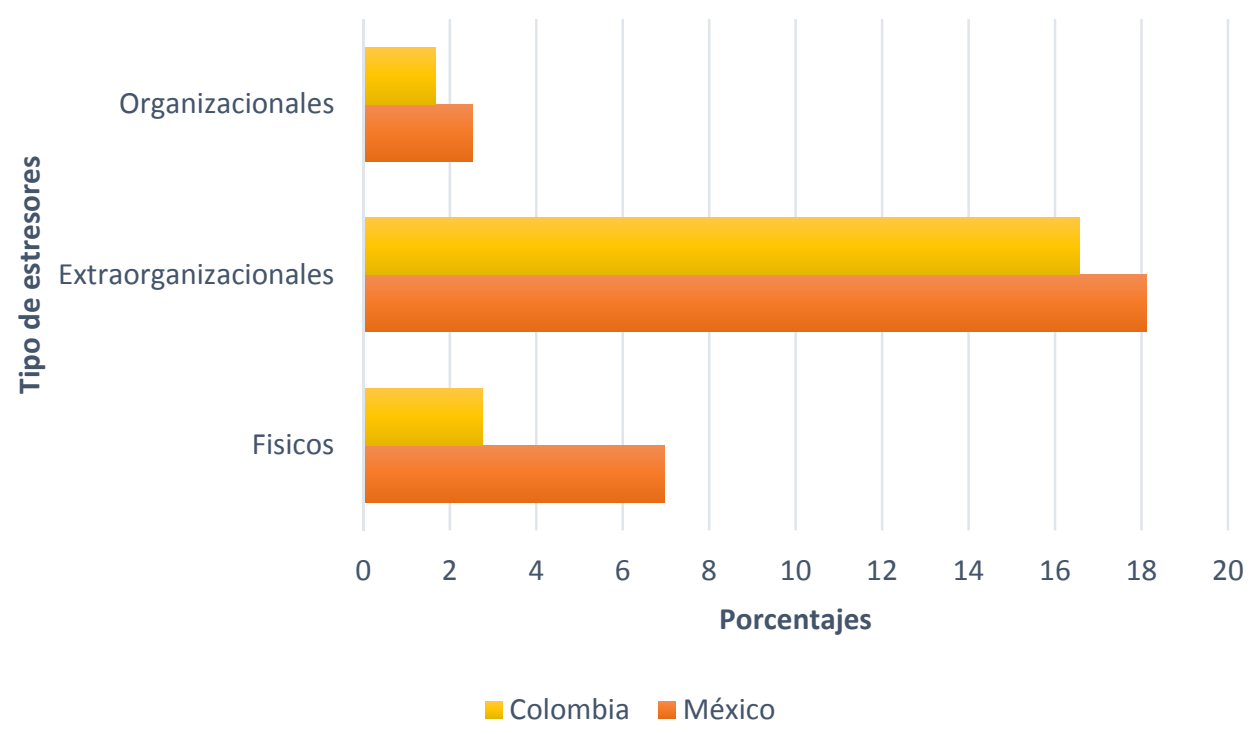

Figura 5. Tipos de estresores causantes de estrés.

Fuente: Elaboración propia.

En la figura 6, se presentan en porcentajes los tipos de estresores referenciados por los integrantes de la muestra que tienen niveles de estrés grave.

Un hallazgo interesante es que en ambas muestras predominan los factores extraorganizacionales, encontrando mayor prevalencia en Colombia (69\%) en relación con México (56\%), en tanto que esta relación se invierte en lo relativo a estresores físicos donde México presenta $28 \%$ y Colombia un $19 \%$, encontrando que en lo relativo a los organizacionales México (16\%) está ligeramente arriba de Colombia (12\%).

- Fisico Extraorganizacional $\quad$ Organizacional

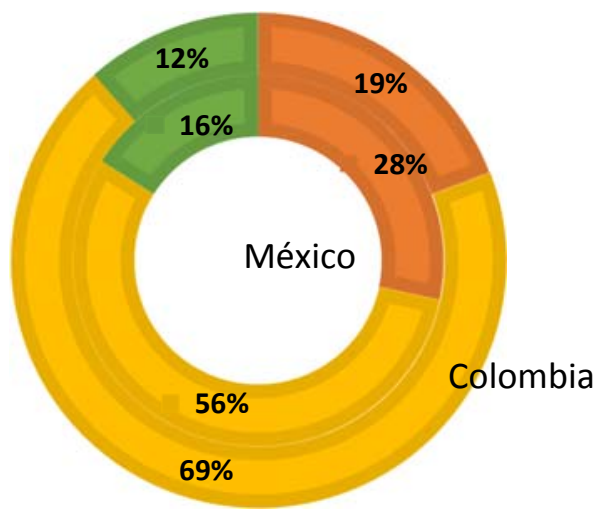

Figura 6. Tipo de estresores presentes en las personas con estrés grave.

Fuente: Elaboración propia. 
ESTUDIO COMPARATIVO MÉXICO-COLOMBIA PARA CONOCER LOS PRINCIPALES FACTORES DE ESTRÉS LABORAL

En la figura 7 , se encontró que el $42.4 \%$ de la muestra de México ha consumido algún tipo de medicamento para la ansiedad y/o depresión, de este porcentaje total tenemos que el 58.2\% corresponde a los que sufren de estrés grave, mientras que en Colombia el $42 \%$ son los que han consumido medicamentos para estas mismas patología, correspondiendo al $42.8 \%$ de los que sufren de estrés grave; esto resulta importante debido a que dentro de los efectos comunes del estrés en el estado de ánimo se encuentran la ansiedad y la depresión (Mayo Clinic, 2018).

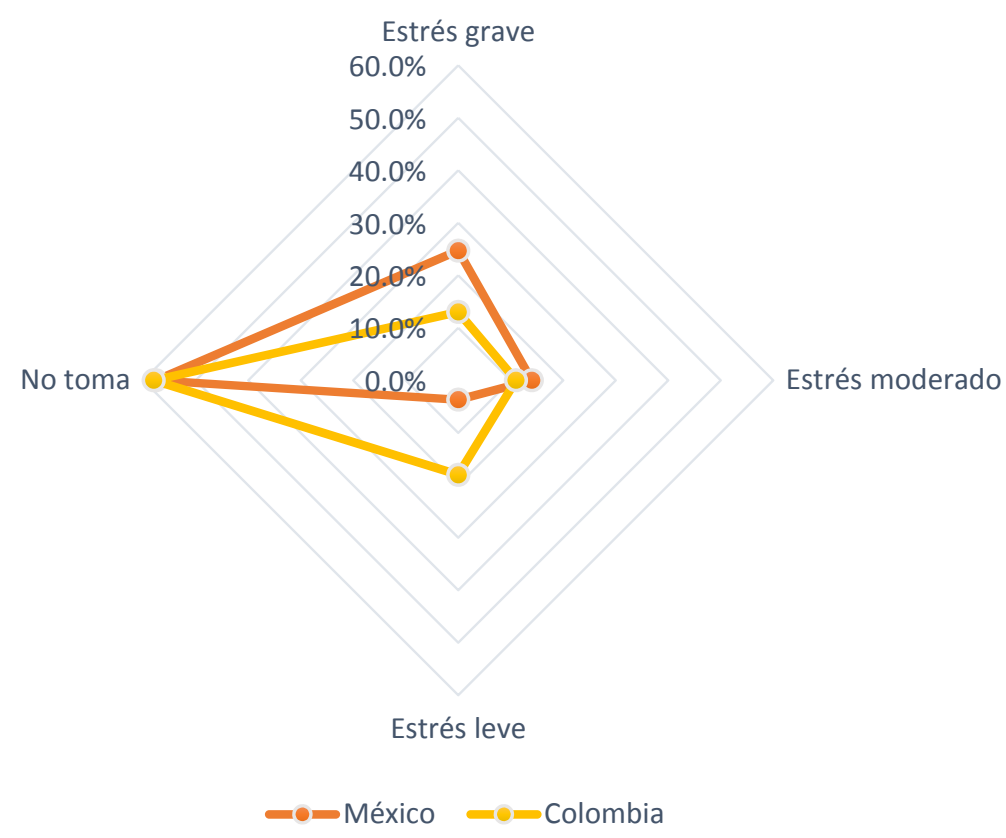

Figura 7. Consume medicamento para ansiedad ylo depresión.

Fuente: Elaboración propia.

\section{CONCLUSIONES}

Considerando los datos obtenidos en la muestra seleccionada es factible concluir:

En lo relativo a los niveles de estrés, se pudo observar que el estrés moderado es el que se presenta en forma más recurrente, siendo mayor su presencia en Colombia (65.74\%) en comparación con México (47.08\%), en este orden de ideas se encontró que en lo relativo al estrés grave ambos países presentan niveles similares (Colombia 21.54\% y México 22.56\%).

En lo relativo a los tipos de estresores identificados, predominaron los extraorganizacionales, donde México (18.11\%) se presentó en forma ligeramente superior a Colombia (16.57\%), al igual 
ESTUDIO COMPARATIVO MÉXICO-COLOMBIA PARA CONOCER LOS PRINCIPALES FACTORES DE ESTRÉS LABORAL

que en los organizaciones (México 2.53\% y Colombia 1.66\%) y en los físicos México 6.96\% presenta un nivel de mayor incidencia en relación con Colombia 2.76\%.

Al realizar una revisión a detalle de los integrantes de la muestra que presentaron estrés identificado como grave, se encontró que el comportamiento de la muestra resulta similar encontrando nuevamente que México presenta mayores incidencias (69.30\%) con respecto a Colombia (55.60\%) en lo relativo a los extraorganizaciones, esta condición se mantiene con los organizaciones (México 16\% y Colombia 11.50\%) y con los físicos (México $28.40 \%$ y Colombia $19.20 \%)$.

Dentro de las consecuencias del estrés se encuentran los trastornos de depresión y ansiedad y en ocasiones estos trastornos se pueden acompañar de abuso de sustancias como son el alcohol, tabaco y sustancias ilegales, por lo que resulta de gran preocupación social estos trastornos, es importante mencionar que la Organización Mundial de la Salud (OMS) ha estimado que la depresión tiene una carga importante socioeconómica y se estimó que en 2010 este trastorno le costó unos 92.000 millones de euros a Europa (Sociedad Española para el Estudio de la Ansiedad y el Estrés - SEAS, 2015). Es claro que un gran porcentaje de la muestra está sufriendo de estos trastornos ya que están consumiendo medicamentos para tratarlos y como se va agravando el estrés el consumo es más alto, por lo que resulta importante combatir el estrés no solo por los costos que causa a nivel organizacional sino también por los efectos a salud que son cada vez más notorios.

\section{REFERENCIAS BIBLIOGRÁFICAS}

American Psychological Association. (2018). Comprendiendo el estrés crónico. Recuperado de https://www.apa.org/centrodeapoyo/estres-cronico.aspx

García, A. (2003). Discriminación de factores de estrés laboral entre ejecutivos y personal operativo en una micro empresa del Valle de México (Tesis de maestría) Secretaría del Trabajo y Previsión Social. México.

Greenspan, F. (1993). Endocrinología básica y clínica. México: El manual moderno.

Instituto Mexicano del Seguro Social. (2018). Estrés Laboral. Recuperado de http://www.imss.gob.mx/salud-en-linea/estres-laboral 
ESTUDIO COMPARATIVO MÉXICO-COLOMBIA PARA CONOCER LOS PRINCIPALES FACTORES DE ESTRÉS LABORAL

Mayo Clinic. (2018). Síntomas de estrés: consecuencias en tu cuerpo y en tu conducta. Recuperado de https://www.mayoclinic.org/es-es/healthy-lifestyle/stress-management/indepth/stress-symptoms/art-20050987

Organización Internacional del Trabajo. (2016). Estres en el trabajo. Ginebra. Recuperado de https://www.ilo.org/public/libdoc/ilo/2016/490658.pdf

Papalia, D. y Wendkos Olds, S. (1988). Psicología. México: McGraw Hill.

Rojas, R. (09 de 04 de 2017). México continúa en primer lugar a nivel mundial en estrés laboral: OMS. Obtenido de https://saludiario.com/mexico-continua-en-primer-lugar-a-nivelmundial-en-estres-laboral-oms/

Sociedad Española para el Estudio de la Ansiedad y el Estrés - SEAS. (2015). Depresión en el ámbito laboral - Informe de la World Federation for Mental Health. Recuperado de http://www.ansiedadyestres.org/content/depresi\%C3\%B3n-en-el-\%C3\%A1mbito-laboral\%E2\%80\%93-informe-de-la-world-federation-mental-health 